\title{
Identification of spin-triplet superconductivity through a helical-chiral phase transition in $\mathrm{Sr}_{2} \mathrm{RuO}_{4}$ thin films
}

\author{
S. Ikegaya, ${ }^{1}$ K. Yada, ${ }^{2}$ Y. Tanaka, ${ }^{2}$ S. Kashiwaya,${ }^{2}$ Y. Asano, ${ }^{3}$ and D. Manske ${ }^{1}$ \\ ${ }^{1}$ Max-Planck-Institut für Festkörperforschung, Heisenbergstrasse 1, D-70569 Stuttgart, Germany \\ ${ }^{2}$ Department of Applied Physics, Nagoya University, Nagoya 464-8603, Japan \\ ${ }^{3}$ Department of Applied Physics, Hokkaido University, Sapporo 060-8628, Japan
}

(Received 31 December 2019; revised manuscript received 20 April 2020; accepted 6 May 2020; published 1 June 2020)

\begin{abstract}
Despite much effort over the past two decades, the pairing symmetry of a $\mathrm{Sr}_{2} \mathrm{RuO}_{4}$ superconductor is still unclear. Motivated by the recent rapid progress in fabrication techniques for $\mathrm{Sr}_{2} \mathrm{RuO}_{4}$ thin films, we propose a promising strategy for identifying the spin-triplet superconductivity in the thin-film geometry by employing an antisymmetric spin-orbit-coupling potential and a Zeeman potential due to an external magnetic field. We demonstrate that a spin-triplet superconducting thin film undergoes a phase transition from a helical state to a chiral state by increasing the applied magnetic field. This phase transition is accompanied by a drastic change in the property of surface Andreev bound states. As a consequence, the helical-chiral phase transition, which is unique to the spin-triplet superconductors, can be detected through a sudden change in a tunneling conductance spectrum of a normal-metal/superconductor junction. Importantly, our proposal is constructed by combining fundamental and rigid concepts regarding the physics of spin-triplet superconductivity.
\end{abstract}

DOI: 10.1103/PhysRevB.101.220501

Introduction. Since 1994, great attention has been drawn to $\mathrm{Sr}_{2} \mathrm{RuO}_{4}$ because it is a leading candidate material for spin-triplet superconductors [1-3]. Actually, on the basis of a number of experiments [4-10] and theories [11-14], $\mathrm{Sr}_{2} \mathrm{RuO}_{4}$ has been believed to exhibit spin-triplet chiral $p$-wave superconductivity with broken time-reversal symmetry $[15,16]$. However, very recent experiments of a nuclear-magneticresonance Knight shift at oxide sites [17,18] seem to be inconsistent with this scenario, and rather suggest the realization of spin-singlet superconductivity in this compound [19]. Such stalemate situation requires us to propose a different way of identifying the spin-triplet superconductivity. In this Rapid Communication, we show that the recent rapid development in fabrication techniques for $\mathrm{Sr}_{2} \mathrm{RuO}_{4}$ thin films enables us to shed some light on this issue.

An essential character of a spin-triplet superconductor is that its order parameter is described by a three-component vector, $\boldsymbol{d}$ vector, reflecting a spin degree of freedom in spin-triplet Cooper pairs. Therefore, in essence, evidence of spin-triplet superconductivity is provided from observations of unique phenomena in the presence of the $\boldsymbol{d}$ vector. A primary factor interacting with the $\boldsymbol{d}$ vector is magnetic potentials, such as Zeeman potentials and exchange potentials. Accordingly, previous studies so far have mainly focused on phenomena of spin-triplet superconductors in the presence

Published by the American Physical Society under the terms of the Creative Commons Attribution 4.0 International license. Further distribution of this work must maintain attribution to the author(s) and the published article's title, journal citation, and DOI. Open access publication funded by the Max Planck Society. of magnetism, such as the temperature-independent spin susceptibility [4-6] and the long-range proximity effect in ferromagnet/superconductor junctions [20-23]. However, at present, the conclusive experimental evidence for the spin-triplet superconductivity has not yet been observed. Substantial progress in this research field arises from the fabrication techniques for $\mathrm{Sr}_{2} \mathrm{RuO}_{4}$ thin films [24-29]. This movement enables us to employ a different factor for the identification of the spin-triplet superconductivity: antisymmetric spin-orbit-coupling (ASOC) potentials due to broken inversion symmetry.

In this Rapid Communication, we study the gap function and transport property of a spin-triplet superconducting thin film that coexists with both an ASOC potential and a Zeeman potential due to an external in-plane magnetic field. We first demonstrate that the spin-triplet superconducting thin film can show a phase transition from helical to chiral spin-triplet superconducting states by increasing the magnetic field. This phase transition is essentially due to the characteristic nature of the $\boldsymbol{d}$ vector and is never expected in spin-singlet superconductors. Then, we show that this phase transition is detected through a drastic change in the tunneling conductance spectrum of normal-metal/superconductor (NS) junctions. As we discuss later, our proposal is constructed of a combination of general and rigid concepts regarding the physics of spin-triplet superconductivity. Consequently, we indicate a promising strategy for identifying the spin-triplet superconductivity in $\mathrm{Sr}_{2} \mathrm{RuO}_{4}$ thin films.

Helical-chiral phase transition. In this Rapid Communication, for simplicity, we focus only on the $\gamma$ band of $\mathrm{Sr}_{2} \mathrm{RuO}_{4}$. We note that a number of previous studies have suggested that the $\gamma$ band plays the dominant role for the superconductivity [2,30-32]. We describe the superconducting states by the following two-dimensional single-band mean-field 
Hamiltonian:

$$
\begin{aligned}
H= & \sum_{\boldsymbol{k}} \sum_{\alpha, \beta}\left(\xi_{\boldsymbol{k}} \hat{\sigma}_{0}+\boldsymbol{g}_{\boldsymbol{k}} \cdot \hat{\boldsymbol{\sigma}}+\boldsymbol{V} \cdot \hat{\boldsymbol{\sigma}}\right)_{\alpha \beta} c_{\boldsymbol{k} \alpha}^{\dagger} c_{\boldsymbol{k} \beta} \\
& +\frac{1}{2} \sum_{\boldsymbol{k}} \sum_{\alpha, \beta}\left[\Delta_{\boldsymbol{k}, \alpha \beta} c_{\boldsymbol{k} \alpha}^{\dagger} c_{-\boldsymbol{k} \beta}^{\dagger}+\text { H.c. }\right] \\
& -\frac{1}{2} \sum_{\boldsymbol{k}} \sum_{\alpha, \beta} \Delta_{\boldsymbol{k}, \alpha \beta}\left\langle c_{\boldsymbol{k} \alpha}^{\dagger} c_{-\boldsymbol{k} \beta}^{\dagger}\right\rangle,
\end{aligned}
$$

where $c_{k \alpha}^{\dagger}\left(c_{k \alpha}\right)$ is the creation (annihilation) operator of an electron with momentum $\boldsymbol{k}$ and spin $\alpha$, the Pauli matrices in spin space are given by $\hat{\sigma}=\left(\hat{\sigma}_{x}, \hat{\sigma}_{y}, \hat{\sigma}_{z}\right)$, and the $2 \times 2$ unit matrix is denoted by $\hat{\sigma}_{0}$. The kinetic energy of an electron measured from the chemical potential $\mu$ is given by

$$
\xi_{k}=-2 t\left(\cos k_{x}+\cos k_{y}\right)-4 t^{\prime} \cos k_{x} \cos k_{y}-\mu,
$$

with $t$ and $t^{\prime}$ representing the nearest-neighbor and nextnearest-neighbor hopping integral, respectively. To reproduce the Fermi surface of the $\gamma$ band, in what follows, we set $t=$ $1.0, t^{\prime}=0.395$, and $\mu=1.5[12,13]$. The ASOC potential is described by the $\boldsymbol{g}$ vector, $\boldsymbol{g}_{\boldsymbol{k}}=-\boldsymbol{g}_{-\boldsymbol{k}}$. Although the momentum dependence of the $\boldsymbol{g}$ vector in real systems may be more complicated, for simplicity, we use the conventional Rashbatype spin-orbit-coupling potential $\boldsymbol{g}_{\boldsymbol{k}}=\lambda\left(\sin k_{y},-\sin k_{x}, 0\right)$. Even so, as we discuss later, the validity of our proposal is insensitive to the detailed structure of $\boldsymbol{g}_{\boldsymbol{k}}$. The Zeeman potential due to an externally applied in-plane magnetic field is $\boldsymbol{V}=\left(V_{x}, V_{y}, 0\right)$. For simplicity, we ignore the orbital pairbreaking effect due to the in-plane magnetic field, which is strongly suppressed in the thin-film limit. The pair potential is represented by $\Delta_{k, \alpha \beta}$. Within the weak-coupling mean-field theory, the pair potential is determined by the gap equation

$$
\Delta_{\boldsymbol{k}, \alpha \beta}=\sum_{\boldsymbol{k}^{\prime}} \sum_{\gamma, \delta} g_{\alpha \beta \gamma \delta}\left(\boldsymbol{k}, \boldsymbol{k}^{\prime}\right)\left\langle c_{-\boldsymbol{k}^{\prime} \gamma} c_{\boldsymbol{k}^{\prime} \delta}\right\rangle,
$$

where $g_{\alpha \beta \gamma \delta}\left(\boldsymbol{k}, \boldsymbol{k}^{\prime}\right)$ is the effective attractive interaction. To reproduce a spin-triplet odd-parity superconductivity, we employ a standard phenomenological attractive interaction $[33,34]$,

$$
g_{\alpha \beta \gamma \delta}\left(\boldsymbol{k}, \boldsymbol{k}^{\prime}\right)=g_{0}\left[\Phi_{x}(\boldsymbol{k}) \Phi_{x}\left(\boldsymbol{k}^{\prime}\right)+\Phi_{y}(\boldsymbol{k}) \Phi_{y}\left(\boldsymbol{k}^{\prime}\right)\right],
$$

for $\left|\xi_{\boldsymbol{k}}\right|,\left|\xi_{\boldsymbol{k}^{\prime}}\right| \leqslant \epsilon_{c}$, and $g_{\alpha \beta \gamma \delta}\left(\boldsymbol{k}, \boldsymbol{k}^{\prime}\right)=0$, for $\left|\xi_{\boldsymbol{k}}\right|,\left|\xi_{\boldsymbol{k}^{\prime}}\right|>\epsilon_{c}$, where we assume that the attractive interaction acts only for the electrons having kinetic energy in the range of $-\epsilon_{c} \leqslant$ $\xi_{k} \leqslant \epsilon_{c}$. The pairing functions have odd-parity symmetry as $\Phi_{x}(\boldsymbol{k})=-\Phi_{x}(-\boldsymbol{k})$ and $\Phi_{y}(\boldsymbol{k})=-\Phi_{y}(-\boldsymbol{k})$. In addition, we assume that the attractive interaction does not depend on the spin. In the absence of the ASOC and Zeeman potential, it has been shown that under the $D_{4 h}$ point-group symmetry of $\mathrm{Sr}_{2} \mathrm{RuO}_{4}$, the gap equation in Eq. (2) has sixfold degenerate solutions such as the four helical states $\boldsymbol{d}_{\boldsymbol{k}}=\left[\Phi_{x}(\boldsymbol{k}), \pm \Phi_{y}(\boldsymbol{k}), 0\right]$ and $\boldsymbol{d}_{\boldsymbol{k}}=\left[\Phi_{y}(\boldsymbol{k}), \pm \Phi_{x}(\boldsymbol{k}), 0\right]$, and the two chiral states, $\boldsymbol{d}_{\boldsymbol{k}}=\left[0,0, \Phi_{y}(\boldsymbol{k}) \pm i \Phi_{x}(\boldsymbol{k})\right]$ [35]. In a real $\mathrm{Sr}_{2} \mathrm{RuO}_{4}$, the atomic spin-orbit coupling lifts the degeneracy of these six spin-triplet superconducting states. In the single-band model, such effect is effectively reproduced by a spin dependence of the attractive interaction [36]. However, the previous theoretical $[14,37]$ and experimental $[6,7]$ studies suggest that the lifting of degeneracy is very small in the bulk, where the splitting in transition temperature $T_{c}$ is estimated to be less than $0.01 T_{c}$ [36]. Therefore, here we ignore the (a) NN pairing dominant

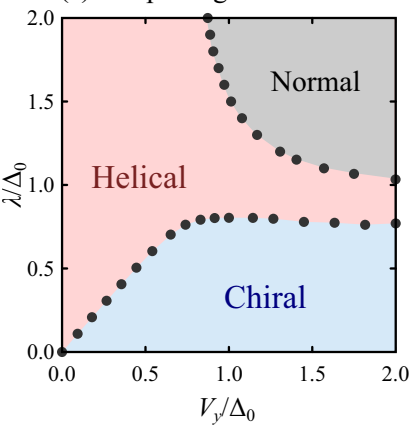

(b) NNN pairing dominant

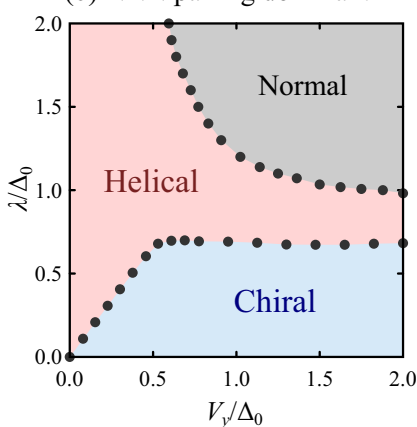

FIG. 1. Phase diagram as a function of the magnitude of ASOC potential $\lambda$ and Zeeman potential. The magnetic field is applied to the $y$ direction as $\boldsymbol{V}=\left(0, V_{y}, 0\right)$. We consider the (a) NN pairing dominant case $(\eta=0.5)$ and (b) NNN pairing dominant case $(\eta=2.0)$.

spin dependence of the attractive interaction for simplicity. Although the substantial form of $\Phi_{x(y)}(\boldsymbol{k})$ is still under discussion, on the basis of several microscopic theories [12,3840], we consider an odd-parity superconductivity within the next-nearest-neighbor pairing as

$$
\Phi_{x(y)}(\boldsymbol{k})=\left[\sin k_{x(y)}-\eta \cos k_{y(x)} \sin k_{x(y)}\right] / \Phi_{0},
$$

where the first term and second term represent the nearestneighbor $(\mathrm{NN})$ pairing and next-nearest-neighbor (NNN) pairing, respectively. We normalize $\Phi_{x(y)}(\boldsymbol{k})$ by $\Phi_{0}$ so that the maximum value of $\Phi_{x(y)}(\boldsymbol{k})$ becomes unity. Since several theories suggest that the NNN pairing becomes dominant [38-42], we consider both the NN pairing dominant case $(\eta=0.5)$ and NNN pairing dominant case $(\eta=2.0)$. By substituting Eq. (3) into Eq. (2), we find that the pair potential is given in the form of $\Delta_{k, \alpha \beta}=\left[\boldsymbol{d}_{\boldsymbol{k}} \cdot \hat{\boldsymbol{\sigma}}\left(i \hat{\sigma}_{y}\right)\right]_{\alpha \beta}$, where the $v$ $(=x, y, z)$ component of the $\boldsymbol{d}$ vector is represented by

$$
d_{\nu}(\boldsymbol{k})=\Delta_{0}\left[X_{\nu} \Phi_{x}(\boldsymbol{k})+Y_{\nu} \Phi_{y}(\boldsymbol{k})\right],
$$

with $X_{v}$ and $Y_{v}$ being the numerical coefficients determined by the gap equation in Eq. (2). To obtain $X_{v}$ and $Y_{v}$, we solve the gap equation by using an iterative method. We show the detailed calculations for solving the gap equation in the Supplemental Material [43]. In what follows, we focus on the gap function at zero temperature. The magnitude of attractive interaction $g_{0}$ is determined so that the amplitude of the pair potential at $\boldsymbol{g}_{\boldsymbol{k}}=\boldsymbol{V}=0$ becomes $\Delta_{0}=0.001$. The cutoff energy $\epsilon_{c}$ is fixed to $10 \Delta_{0}$.

In Figs. 1(a) and 1(b), we show the phase diagrams obtained from the gap equation as a function of the magnitude of the ASOC potential $\lambda$ and Zeeman potential $|\boldsymbol{V}|$. We consider the NN pairing dominant case $(\eta=0.5)$ and NNN pairing dominant case $(\eta=2.0)$ in Figs. $1(\mathrm{a})$ and $1(\mathrm{~b})$, respectively. We apply the magnetic field to the $y$ direction as $\boldsymbol{V}=\left(0, V_{y}, 0\right)$. In both cases, for small Zeeman potentials, we obtain the helical state where the $\boldsymbol{d}$ vector is approximately given as

$$
\boldsymbol{d}_{\boldsymbol{k}}^{h} \propto\left[\Phi_{y}(\boldsymbol{k}),-\delta \Phi_{x}(\boldsymbol{k}), 0\right],
$$

with $\delta$ being a real number in the range of $0<\delta<1$. For small ASOC potentials and large Zeeman potentials, in both Figs. 1(a) and 1(b), we find the chiral state approximately 
described by

$$
\boldsymbol{d}_{\boldsymbol{k}}^{c} \propto\left[\Phi_{y}(\boldsymbol{k}) \pm i \Phi_{x}(\boldsymbol{k}), 0,0\right]
$$

where the $\boldsymbol{d}$ vector satisfies $\boldsymbol{d}_{\boldsymbol{k}}^{c} \perp \boldsymbol{V}$. In contrast to the bulk chiral states, $\boldsymbol{d}_{\boldsymbol{k}}^{c}$ is pinned in the basal plane. For the large ASOC and Zeeman potentials, we obtain the normal states with $\boldsymbol{d}_{\boldsymbol{k}}=0$. The detailed structure of the $\boldsymbol{d}$ vector is shown in the Supplemental Material [43]. At the phase boundary from the helical phase to the chiral phase, the $\boldsymbol{d}$ vector suddenly changes from $\boldsymbol{d}_{\boldsymbol{k}}^{h}$ to $\boldsymbol{d}_{\boldsymbol{k}}^{c}$ (see, also, the Supplemental Material [43]). This suggests that the helical-chiral phase transition is the first order. At the phase boundary from the helical phase to the normal phase, the amplitude of $\boldsymbol{d}_{\boldsymbol{k}}^{h}$ suddenly drops to zero. This implies that the helical state undergoes a first-order phase transition to the normal state. Even so, in analogy with spin-singlet superconductors [44-46], there is a possibility that a Fulde-Ferrell-Larkin-Ovchinnikov (FFLO) state appears in the vicinity of the first-order phase boundary between the superconducting state and the normal state. However, in this Rapid Communication, we focus on the phase transition from the helical state to the chiral state, and the possibility for the FFLO phase in the vicinity of the normal phase is beyond the scope of this work. Importantly, the presence of the helical-chiral phase transition is well understood by the following two generic features of the $\boldsymbol{d}$ vector:

(i) The pair-breaking effect of an ASOC potential $g$ damages the component of the $\boldsymbol{d}$ vector perpendicular to $\boldsymbol{g}$ [47]. Thus, in the presence of the ASOC potential, helical states are more energetically favorable than chiral states because helical states can have a larger inner product of $\left|\boldsymbol{d}_{\boldsymbol{k}} \cdot \boldsymbol{g}_{\boldsymbol{k}}\right|$ to optimize the condensation energy [38,47-49].

(ii) The paramagnetic pair-breaking effect of a Zeeman potential $\boldsymbol{V}$ damages the component of the $\boldsymbol{d}$ vector parallel to $\boldsymbol{V}$. Thus, the chiral states satisfying $\boldsymbol{d} \perp \boldsymbol{V}$ are energetically favorable in the presence of the Zeeman potential because they are completely free from the paramagnetic pair-breaking effect [41,50,51].

The realization of the helical state of $\boldsymbol{d}_{\boldsymbol{k}}^{h}$ for small Zeeman potentials is mainly explained by feature (i). Namely, the helical state appears for small Zeeman potentials to minimize the dominant pair-breaking effect from the ASOC potential. The suppression in the $x$ component of $\boldsymbol{d}_{\boldsymbol{k}}^{h}$ characterized by $\delta$ is due to the paramagnetic pair-breaking effect discussed in feature (ii). The realization of the chiral states of $\boldsymbol{d}_{\boldsymbol{k}}^{c}$ for large Zeeman potentials is naturally understood from feature (ii). The in-plane chiral states of $\boldsymbol{d}_{\boldsymbol{k}}^{c}$ are more stable than the out-of-plane chiral states, $\boldsymbol{d}_{\boldsymbol{k}}=\left[0,0, \Phi_{y}(\boldsymbol{k}) \pm i \Phi_{x}(\boldsymbol{k})\right]$, because the in-plane chiral states have a larger inner product of $\left|\boldsymbol{d}_{\boldsymbol{k}} \cdot \boldsymbol{g}_{\boldsymbol{k}}\right|$ discussed in feature (i). Features (i) and (ii) are the rigid concepts irrelevant to the details of the model. Actually, it has been confirmed that feature (i) $[38,48,49]$ and feature (ii) $[50,51]$ are valid even with the multiband models for $\mathrm{Sr}_{2} \mathrm{RuO}_{4}$. We also confirm that the helical-chiral phase transition occurs even when we employ a more realistic ASOC potential discussed in Ref. [48]. Moreover, in principle, the amplitude of ASOC potentials can be tuned by changing the substrate, by fabricating capping layers, or by applying gate voltages. Therefore, we can expect that spin-triplet superconducting thin films show the helical-chiral phase transition in experiments.
Although the orbital pair-breaking effect due to the inplane magnetic field is suppressed in the thin-film geometry, it may exist in reality. Even so, the orbital pair-breaking effect damages the helical and chiral states in the same manner, where the magnetic field in the $y$ direction suppresses the components of the $\boldsymbol{d}$ vector depending on $k_{x}$ in both the helical and chiral states. Therefore, the difference in the condensation energy between the helical and chiral states, which is generated only from the spin part, is retained even in the presence of the orbital pair-breaking effect. As a consequence, although the orbital pair-breaking effect may shrink the superconducting region in the phase diagrams, we can still expect the helical-chiral phase transition in the thin film of $\mathrm{Sr}_{2} \mathrm{RuO}_{4}$.

Signature in tunneling spectroscopy. Next, to approach the detection of the helical-chiral phase transition in experiments, we study the differential conductance in a twodimensional normal-metal/spin-triplet superconductor (NS) junction. We assume that the NS junction consists of the semi-infinite normal-metal segment located for $x<x_{0}$ and the semi-infinite spin-triplet superconducting segment located for $x \geqslant x_{0}$, where the periodic boundary condition is applied to the direction parallel to the junction interface (i.e., the $y$ direction). We describe the normal segment by setting $\Delta_{0}=0$. To describe the superconducting segment, we use the $\boldsymbol{d}$ vector obtained from the gap equation in Eq. (2). The hopping integrals between the normal and superconducting segments are chosen as $t=0.05$ and $t^{\prime}=0.0$ to describe a low-transparency junction. The Hamiltonian used for calculating the differential conductance is explicitly shown in the Supplemental Material [43]. We calculate the differential conductance $G_{\mathrm{NS}}$ based on the formula [52-54]

$$
G_{\mathrm{NS}}(e V)=\frac{e^{2}}{h} \sum_{\zeta, \zeta^{\prime}}\left[\delta_{\zeta, \zeta^{\prime}}-\left|r_{\zeta, \zeta^{\prime}}^{e e}\right|^{2}+\left|r_{\zeta, \zeta^{\prime}}^{h e}\right|^{2}\right]_{e V=E}
$$

where $r_{\zeta, \zeta^{\prime}}^{e e}$ and $r_{\zeta, \zeta^{\prime}}^{\text {he }}$ denote the normal and Andreev reflection coefficients at the energy $E$, respectively. The indices $\zeta$ and $\zeta^{\prime}$ label the outgoing and incoming channel, respectively. These reflection coefficients are calculated by using the lattice Green's function techniques $[55,56]$. The results are normalized by the normal conductance $G_{\mathrm{N}}$, which is calculated by setting $\boldsymbol{V}=\boldsymbol{d}_{\boldsymbol{k}}=0$.

In Figs. 2(a) and 2(b), we show the differential conductance of the NS junction as a function of the bias voltage $e V$ with $\lambda=0.5 \Delta_{0}$ and $\boldsymbol{V}=\left(0, V_{y}, 0\right)$. We consider the NN pairing dominant case $(\eta=0.5)$ and NNN pairing dominant case $(\eta=2.0)$ in Figs. 2(a) and 2(b), respectively. With $\lambda=$ $0.5 \Delta_{0}$, the phase boundary between the helical and chiral phase is located at $V_{y}=0.441 \Delta_{0}\left(0.373 \Delta_{0}\right)$ for $\eta=0.5$ $(\eta=2.0)$. We choose $V_{y}$ very close to the phase boundary: $V_{y}=V_{c} \pm 0.001 \Delta_{0}$ for the helical (chiral) state. As shown in Figs. 2(a) and 2(b), the conductance spectra for the helical states (red line) show the U-shaped structures [57]. Nevertheless, when the helical states undergo the phase transition to the chiral states, the conductance spectra show the sudden enhancement in low-bias voltages (blue line), whereas the steep zero-bias dip is found for $\eta=0.5$. The sudden change in the tunneling conductance implies that the properties of surface Andreev bound states (ABSs) are changed drastically 
(a) NN pairing dominant

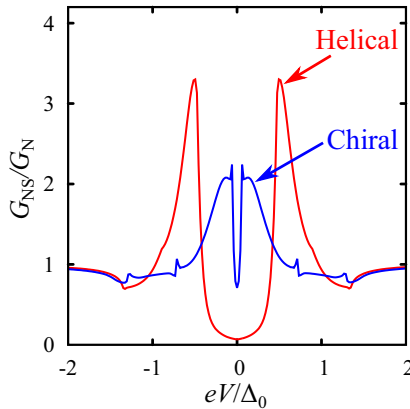

(b) NNN pairing dominant

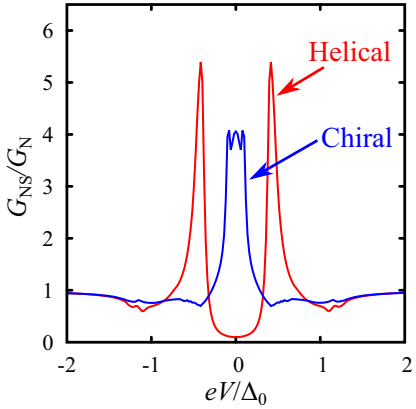

FIG. 2. Differential conductance as a function of the bias voltage with (a) $\eta=0.5$ and (b) $\eta=2.0$. We choose $V_{y}$ very close to the phase boundary: $V_{y}=V_{c} \pm 0.001 \Delta_{0}$ for the helical (chiral) state, with $V_{c}$ representing the critical magnitude of the Zeeman potential at $\lambda=0.5 \Delta_{0}$.

through the phase transition. To confirm this statement, we also calculate the surface density of states (DOS) for the semi-infinite superconductor by using the formula $\rho_{k_{y}}(E)=$ $-\operatorname{Im}\left[\operatorname{Tr} G_{k_{y}}\left(x_{0}, x_{0}, E+i \gamma\right)\right] / \pi$, with $G_{k_{y}}\left(x, x^{\prime}, E\right)$ representing the Green's function with momentum $k_{y}$ parallel to the surface. $\gamma$ denotes the small imaginary part added to the energy. Tr means the trace for spin and Nambu space of the Green's function. To calculate the surface DOS, we remove the semi-infinite normal segment located for $x<x_{0}$. The small imaginary part of the energy in the Green's function is chosen as $\gamma=10^{-3} \Delta_{0}$. In Figs. 3(a) $-3(\mathrm{~d})$, we show the surface DOS as a function of the energy and momentum parallel to the surface. The white lines denote the lowest bulk energy for each $k_{y}$, which is obtained by diagonalizing the bulk Hamiltonian. Due to the pair-breaking effect of the ASOC and Zeeman potentials, the bulk superconducting gap partially vanishes in momentum space. For the helical phases, as shown in Figs. 3(a) and 3(b), the ABSs are absent in low energies. In contrast, as shown in Figs. 3(c) and 3(d), we can still find the inner-gap ABSs in the chiral phases. This qualitative difference in the ABSs of the helical phase and that of the chiral phase is related to a generic concept regarding the topological classification [58]:

(iii) A helical (chiral) superconductor in two dimensions can exhibit the surface ABSs characterized by a $\mathbb{Z}_{2}(\mathbb{Z})$ topological invariant. Thus, the ABSs of the helical (chiral) superconductor are intrinsically fragile (robust) against Zeeman potentials breaking time-reversal symmetry.

Strictly speaking, we can no longer employ the topological invariant for the present junction because the bulk superconducting gap is closed. Even so, the absence (presence) of the ABSs in the helical (chiral) phase is well understood by their intrinsic fragility (robustness) against the Zeeman potential. The detailed structure of the conductance spectra depends on the details of the model for the $\mathrm{Sr}_{2} \mathrm{RuO}_{4}$ superconductors [57,59-62]. However, according to the topological concepts (iii) irrelevant to the details of the model, the surface ABSs in the helical phase and that in the chiral phase have distinctively different characters in the presence (a) Helical, $\eta=0.5$

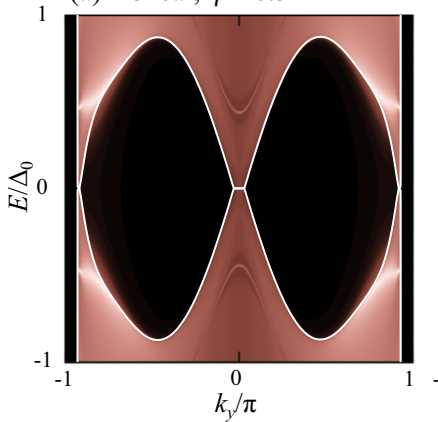

(c) Chiral, $\eta=0.5$

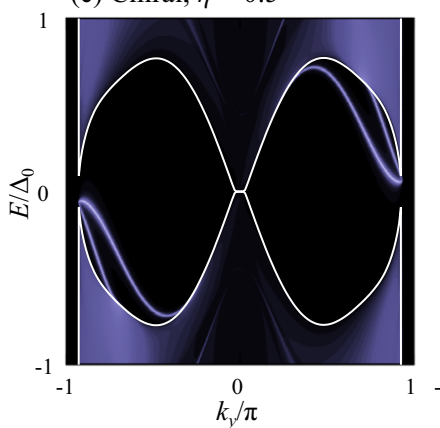

(d) Chiral, $\eta=2.0$

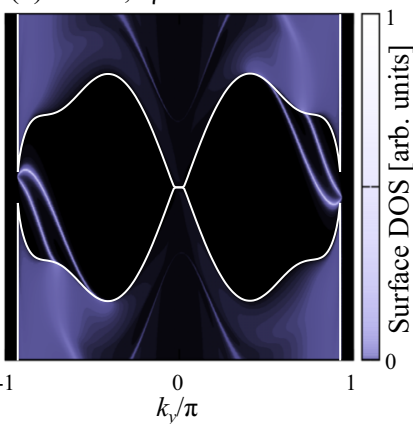

FIG. 3. Surface density of states for the semi-infinite superconductor as a function of the energy and momentum parallel to the surface. For the (a),(b) helical and (c),(d) chiral phases, we use $V_{y}=V_{c} \pm 0.001 \Delta_{0}$. The white lines denote the lowest bulk-energy dispersion obtained by diagonalizing the bulk Hamiltonian.

of the Zeeman potential. Therefore, even in real experiments, we can likely expect that the helical-chiral phase transition can be detected through the drastic change in the tunneling conductance spectrum.

Summary. In conclusion, we demonstrate that spin-triplet superconducting thin films show the helical-chiral phase transition by applying in-plane magnetic fields (see Fig. 1). This phase transition is unique in the presence of the $\boldsymbol{d}$ vector and is intrinsically absent in spin-singlet superconductors. The helical-chiral phase transition can be detected by the sudden change in the conductance spectrum of the NS junction (see Fig. 2), reflecting the drastic change in the properties of the surface ABSs through the phase transition (see Fig. 3). Our proposal is constructed of the combination of the three fundamental and rigid concepts (i)-(iii) irrelevant to the details of the model. Consequently, we propose a promising strategy for identifying the realization of spin-triplet superconductivity in $\mathrm{Sr}_{2} \mathrm{RuO}_{4}$ thin films.

Acknowledgments. We are grateful to S. Yonezawa for fruitful discussions. This work was supported by Grants-inAid from JSPS for Scientific Research on Innovative Areas "Topological Materials Science" (KAKENHI Grants No. JP15H05851, No. JP15H05853, No. JP15H05855, and No. JP15K21717), Scientific Research (B) (KAKENHI Grant No. JP18H01176), and Scientific Research (A) (KAKENHI Grant No. JP20H00131). This work was also supported by JST CREST (Grant No. JPMJCR16F2) and JSPS Core-to-Core program "Oxide Superspin" international network. 
[1] Y. Maeno, H. Hashimoto, K. Yoshida, S. Nishizaki, T. Fujita, J. G. Bednorz, and F. Lichtenberg, Nature (London) 372, 532 (1994).

[2] A. P. Mackenzie and Y. Maeno, Rev. Mod. Phys. 75, 657 (2003).

[3] Y. Maeno, S. Kittaka, T. Nomura, S. Yonezawa, and K. Ishida, J. Phys. Soc. Jpn. 81, 011009 (2012).

[4] J. A. Duffy, S. M. Hayden, Y. Maeno, Z. Mao, J. Kulda, and G. J. McIntyre, Phys. Rev. Lett. 85, 5412 (2000).

[5] K. Ishida, H. Mukuda, Y. Kitaoka, Z. Q. Mao, H. Fukazawa, and Y. Maeno, Phys. Rev. B 63, 060507(R) (2001).

[6] H. Murakawa, K. Ishida, K. Kitagawa, Z. Q. Mao, and Y. Maeno, Phys. Rev. Lett. 93, 167004 (2004).

[7] H. Murakawa, K. Ishida, K. Kitagawa, H. Ikeda, Z. Q. Mao, and Y. Maeno, J. Phys. Soc. Jpn. 76, 024716 (2007).

[8] H. Mukuda, K. Ishida, Y. Kitaoka, K. Miyake, Z. Q. Mao, Y. Mori, and Y. Maeno, Phys. Rev. B 65, 132507 (2002).

[9] J. Jang, D. G. Ferguson, V. Vakaryuk, R. Budakian, S. B. Chung, P. M. Goldbart, and Y. Maeno, Science 331, 186 (2011).

[10] S. Kashiwaya, H. Kashiwaya, H. Kambara, T. Furuta, H. Yaguchi, Y. Tanaka, and Y. Maeno, Phys. Rev. Lett. 107, 077003 (2011).

[11] T. M. Rice and M. Sigrist, J. Phys.: Condens. Matter 7, L643 (1995).

[12] T. Nomura and K. Yamada, J. Phys. Soc. Jpn. 69, 3678 (2000).

[13] T. Nomura and K. Yamada, J. Phys. Soc. Jpn. 71, 404 (2002).

[14] Y. Yanase and M. Ogata, J. Phys. Soc. Jpn. 72, 673 (2003).

[15] G. M. Luke, Y. Fudamoto, K. M. Kojima, M. I. Larkin, J. Merrin, B. Nachumi, Y. J. Uemura, Y. Maeno, Z. Q. Mao, Y. Mori, H. Nakamura, and M. Sigrist, Nature (London) 394, 558 (1998).

[16] J. Xia, Y. Maeno, P. T. Beyersdorf, M. M. Fejer, and A. Kapitulnik, Phys. Rev. Lett. 97, 167002 (2006).

[17] A. Pustogow, Y. Luo, A. Chronister, Y.-S. Su, D. A. Sokolov, F. Jerzembeck, A. P. Mackenzie, C. W. Hicks, N. Kikugawa, S. Raghu, E. D. Bauer, and S. E. Brown, Nature (London) 574, 72 (2019).

[18] K. Ishida, M. Manago, K. Kinjo, and Y. Maeno, J. Phys. Soc. Jpn. 89, 034712 (2020).

[19] H. S. Røising, T. Scaffidi, F. Flicker, G. F. Lange, and S. H. Simon, Phys. Rev. Res. 1, 033108 (2019).

[20] N. Yoshida, Y. Tanaka, J. Inoue, and S. Kashiwaya, J. Phys. Soc. Jpn. 68, 1071 (1999).

[21] T. Hirai, N. Yoshida, Y. Tanaka, J.-i. Inoue, and S. Kashiwaya, J. Phys. Soc. Jpn. 70, 1885 (2001).

[22] B. Kastening, D. K. Morr, D. Manske, and K. Bennemann, Phys. Rev. Lett. 96, 047009 (2006).

[23] M. S. Anwar, S. R. Lee, R. Ishigro, Y. Sugimoto, Y. Tano, S. J. Kang, Y. J. Shin, S. Yonezawa, D. Manske, H. Takayanagi, T. W. Noh, and Y. Maeno, Nat. Commum. 7, 13220 (2016).

[24] Y. Krockenberger, M. Uchida, K. S. Takahashi, M. Nakamura, M. Kawasaki, and Y. Tokura, Appl. Phys. Lett. 97, 082502 (2010).

[25] J. Cao, D. Massarotti, M. E. Vickers, A. Kursumovic, A. D. Bernardo, J. W. A. Robinson, F. Tafuri, J. L. MacManusDriscoll, and M. G. Blamire, Supercond. Sci. Technol. 29, 095005 (2016).

[26] M. Uchida, M. Ide, H. Watanabe, K. S. Takahashi, Y. Tokura, and M. Kawasaki, APL Matter 5, 106108 (2017).
[27] H. P. Mair, J. P. Ruf, N. J. Schreiber, L. Miao, M. L. Grandon, D. J. Baek, B. H. Goodge, J. P. C. Ruff, L. F. Kourkoutis, K. M. Shen, and D. G. Schlom, APL Matter 6, 101108 (2018).

[28] M. Uchida, M. Ide, M. Kawamura, K. S. Takahashi, Y. Kozuka, Y. Tokura, and M. Kawasaki, Phys. Rev. B 99, 161111(R) (2019).

[29] G. Kim, Y. E. Suyolcu, J. Herrero-Martin, D. Putzky, H. P. Nair, J. P. Ruf, N. J. Schreiber, C. Dietl, G. Christiani, G. Logvenov, M. Minola, P. A. van Aken, K. M. Shen, D. G. Schlom, and B. Keimer, Phys. Rev. Mater. 3, 094802 (2019).

[30] K. Deguchi, Z. Q. Mao, H. Yaguchi, and Y. Maeno, Phys. Rev. Lett. 92, 047002 (2004).

[31] K. Deguchi, Z. Q. Mao, and Y. Maeno, J. Phys. Soc. Jpn. 73, 1313 (2004).

[32] T. Nomura and K. Yamada, J. Phys. Soc. Jpn. 71, 1993 (2002).

[33] A. J. Leggett, Rev. Mod. Phys. 48, 357 (1976).

[34] J. Hara and K. Nagai, Prog. Theor. Phys. 76, 1237 (1986).

[35] The nematic $p$-wave states, $\boldsymbol{d}_{\boldsymbol{k}}=\left[0,0, \Phi_{x}(\boldsymbol{k}) \pm \Phi_{y}(\boldsymbol{k})\right]$, are also allowed by the $D_{4 h}$ point-group symmetry. However, the nematic $p$-wave states are typically less favorable than helical and chiral states because of the presence of the gap nodes.

[36] M. Udagawa, Y. Yanase, and M. Ogata, J. Phys. Soc. Jpn. 74, 2905 (2005).

[37] J. F. Annett, B. L. Györffy, G. Litak, and K. I. Wysokiński, Phys. Rev. B 78, 054511 (2008).

[38] Y. Tada, N. Kawakami, and S. Fujimoto, New J. Phys. 11, 055070 (2009).

[39] Q. H. Wang, C. Platt, Y. Yang, C. Honerkamp, F. C. Zhang, W. Hanke, T. M. Rice, and R. Thomale, Europhys. Lett. 104, 17013 (2013).

[40] T. Scaffidi and S. H. Simon, Phys. Rev. Lett. 115, 087003 (2015).

[41] R. P. Kaur, D. F. Agterberg, and H. Kusunose, Phys. Rev. B 72, 144528 (2005).

[42] W. Huang, S. Lederer, E. Taylor, and C. Kallin, Phys. Rev. B 91, 094507 (2015).

[43] See Supplemental Material at http://link.aps.org/supplemental/ 10.1103/PhysRevB.101.220501 for the detailed calculations of the phase diagrams in Fig. 1 and the conductance spectra in Fig. 2. We also discuss the detailed structure of the gap functions.

[44] P. Fulde and R. A. Ferrell, Phys. Rev. 135, A550 (1964).

[45] A. I. Larkin and Yu. N. Ovchinnikov, Zh. Eksp. Teor. Fiz. 47, 1136 (1964) [Sov. Phys. JETP 20, 762 (1965)].

[46] K. Yang and S. L. Sondhi, Phys. Rev. B 57, 8566 (1998).

[47] P. A. Frigeri, D. F. Agterberg, A. Koga, and M. Sigrist, Phys. Rev. Lett. 92, 097001 (2004).

[48] Y. Yanase, J. Phys. Soc. Jpn. 82, 044711 (2013).

[49] M. A. Scheurer, D. F. Agterberg, and J. Schmalian, npj Quantum Mater. 2, 9 (2017).

[50] Y. Yanase, S. Takamatsu, and M. Udagawa, J. Phys. Soc. Jpn. 83, 061019 (2014).

[51] K. Oda, K. K. Tanaka, S. Onari, and M. Ichioka, J. Phys. Soc. Jpn. 88, 064707 (2019).

[52] G. E. Blonder, M. Tinkham, and T. M. Klapwijk, Phys. Rev. B 25, 4515 (1982).

[53] C. Bruder, Phys. Rev. B 41, 4017 (1990). 
[54] S. Kashiwaya and Y. Tanaka, Rep. Prog. Phys. 63, 1641 (2000). [55] P. A. Lee and D. S. Fisher, Phys. Rev. Lett. 47, 882 (1981).

[56] T. Ando, Phys. Rev. B 44, 8017 (1991).

[57] K. Sengupta, H.-J. Kwon, and V. M. Yakovenko, Phys. Rev. B 65, 104504 (2002).

[58] A. P. Schnyder, S. Ryu, A. Furusaki, and A. W. W. Ludwig, Phys. Rev. B 78, 195125 (2008).
[59] M. Yamashiro, Y. Tanaka, and S. Kashiwaya, Phys. Rev. B 56, 7847 (1997).

[60] C. Honerkamp and M. Sigrist, J. Low Temp. Phys. 111, 895 (1998).

[61] S. Wu and K. V. Samokhin, Phys. Rev. B 81, 214506 (2010).

[62] K. Yada, A. A. Golubov, Y. Tanaka, and S. Kashiwaya, J. Phys. Soc. Jpn. 83, 074706 (2014). 